\title{
35 Research Soure

\section{Total saponins from Trillium Tschonoskii Maxim alleviate myocardial ischemia reperfusion injury in rat}

\section{Xiujing Zhang ( $\nabla$ xiujingzhang66@163.com )}

Beijing University of Chinese Medicine Third Affiliated Hospital

\section{Wei Wang}

Beijing University of Chinese Medicine Third Affiliated Hospital

Jie Tang

Beijing Henghe hospital

Qin Xie

Beijing Heng He Hospital

\section{Di Ma}

Beijing University of Chinese Medicine Third Affiliated Hospital

\section{Research}

Keywords: Myocardial ischemia reperfusion injury, Total saponins from Trillium Tschonoskii Maxim, TNFa, Apoptosis, AMPK/Sirt1 signaling pathway

Posted Date: September 2nd, 2020

DOI: https://doi.org/10.21203/rs.3.rs-68498/v1

License: (c) (1) This work is licensed under a Creative Commons Attribution 4.0 International License. Read Full License 


\section{Abstract}

\section{Background}

To investigate the effect and possible mechanisms of total saponins from Trillium Tschonoskii Maxim. (TST) on myocardial ischemia reperfusion injury in rat.

\section{Methods and Results}

Rats were pre-treated with TST in 100 and $200 \mathrm{mg} / \mathrm{kg}$, respectively. After 14 days intragastric administration, the model of myocardial ischemia-reperfusion injury was established by ligation of the left anterior descending coronary artery for $30 \mathrm{~min}$ and then releasing the ligated artery for $120 \mathrm{~min}$. The hemodynamic indexes, anti-oxidation index, and the anti-inflammation factors were detected. Pathological changes in myocardia tissue were observed by H\&E staining. Apoptosis protein expression of caspase 3, 9, 12, AMPK, phosphorylation AMPK ( $p$-AMPK) and Sirt1 was detected by Western blot. Pretreating the rats with TST dramatically decreased the levels of MDA, TNF- $\alpha$, IL- 6 and IL- $1 \beta$, increased the levels of SOD and GSH-Px, and the apoptosis protein expression were all significantly decreased. In addition, the protein expression of $p$-AMPK and Sirt1 were markedly increased in TST pre-treated group. Furthermore, TST pre-treatment also improved the histopathological changes.

\section{Conclusion}

TST protect the myocardium by reducing the levels of inflammation factors, peroxides and cell apoptosis, increasing the anti-oxidase, and improving the pathological changes. The possible mechanism maybe through the activating of the AMPK/Sirt1 signaling pathway.

\section{Background}

Cardiovascular diseases (CVDs) kill 17.9 million people every year, accounting for $31 \%$ of global deaths [1]. According to the latest statistics of WHO, 56.9 million deaths worldwide in 2016, among them, there are 15.2 million deaths caused by ischemic heart disease[1]. Ischemic heart disease has been the main cause of global human death in the past 15 years $[2,3]$. Thus, a certain proportion of ischemiareperfusion injury (IRI) will inevitably occur in the process of treating ischemic heart disease [4]. When the ischemic myocardium recovers the blood perfusion, the structure and function of the myocardium can also be restored. However, because of the combined effect of a variety of complex molecular mechanisms, when some myocardium recovers the perfusion, the myocardial injury becomes more and more serious, resulting in irreversible damage such as myocardial death, which is called myocardial ischemia-reperfusion injury (MIRI) [5-7]. With the development of science and medical treatment, thrombolysis, revascularization, coronary stenting, and coronary artery bypass grafting may cause MIRI. Therefore, how to effectively prevent and treat MIRI has become a hot topic in recent years [8-10]. 
The mechanism of MIRI is very complex, which mainly include oxidative stress, calcium overload, energy metabolism disorder, inflammatory response, mitochondrial cell apoptosis and so on [11-13]. Relevant statistics show that nearly $1 / 4$ of patients cannot provide enough normal myocardium after myocardial ischemia-reperfusion treatment, resulting in serious heart failure [14-16]. Therefore, the mechanism study of blood reperfusion injury has become the focus in the research of myocardial ischemia. Some studies have shown that the protective agent of cardiomyocytes administrated before myocardial ischemia-reperfusion has a significant inhibitory effect on myocardial injury [17-19], which is of great significance for the prevention of MIRI. Therefore, it is very important to find effective drugs for the treatment of MIRI.

Trillium tschonoskii Maxim. (TTM), a plant from Trillocerosaceae family, is locally known as 'Yan Ling Cao', which meaning that it can prolong human life [20,21]. As a traditional Chinese medicinal plant in mid-western part of China, the dried root and rhizome of TTM were traditionally used for the treatment of hypertension, dizziness, neurasthenia, waist leg pain, bleeding, headache, and traumatic haemorrhage [22]. There have reported many literatures of TTM about in vivo animal studies, such as TTM extracts could mitigates D-galactose-induced brain aging of rats [21], attenuates abnormal Tau phosphorylation in rats [20], anti-tumor in non-small cell lung cancer in mice [23, 24], reduce myocardial injury in diabetic rats [25], decrease liver injury in rats [26], and etc. Phytochemical studies revealed that TTM contains many of saponins [27-29], saponin, as the major bioactive component of TTM, were found to possess various bioactivities, for example anti-inflammatory, immune-improving, anti-aging, anti-oxidative, anti-bacterial, anti-virus and anti-tumor properties [30,31]. Although the saponins isolated from TTM showed various bioactivities, the reported pharmacological activities on MIRI were limited.

Furthermore, it was reported that the activation of adenosine $5^{\prime}$-mono phosphate activated protein kinase (AMPK) / silent information regulator 1 (Sirt1) signaling pathway could inhibited MIRI through alleviate inflammation reaction [32-35]. Thus, in this study, we attempted to explore and evaluate the protective effect and potential mechanisms of TST in MIRI in rat. In addition, based on the literature reported about AMPK / Sirt1 signaling pathway on MIRI, here, we also would like to know if TST have the protective effects on MIRI, is it related with AMPK / Sirt1 signaling pathway? The study would provide the experimental evidence for further clinical therapy of MIRI by TST.

\section{Methods}

\section{Materials and reagents}

Superoxide dismutase (SOD, A001-3-2), malondialdehyde (MDA, A003-1-2) and glutathione peroxidase (GSH-Px, A005-1-2) reagents kit were purchased from Nanjing Jiancheng Bioengineering Institute (Nanjing, China). ELISA detection kits of tumor necrosis factor-alpha (TNF- $a$, K1051-100), Interleukin (IL)-6 (K4145-100) and IL-1 $\beta$ (K4796-100) were purchased from BioVision (USA). NF- $K B$ p65 acetyl K310 (ab19870), Sirt1 (ab110304), F4/80 (ab100790) antibodies and TUNEL assay kit (ab66108) were purchased from Abcam (USA). Caspase 3 (9662), caspase 9 (9508), caspase 12 (2202), AMPK (2532) 
and $p$-AMPK (2535) antibody were purchased from cell signaling technology (USA). Horseradish peroxidase (HRP)-conjugated goat anti-mouse IgG and goat anti-rabbit IgG antibodies were provided by Proteintech Group, Inc. (USA). BCA protein assay kit was from Zhongshan Institute of Biotechnology (Beijing, China).

\section{Preparation of TST [36]}

The root and rhizome of TTM was purchased from Enshi Chinese Medicine Co. Ltd. (Enshi, Hubei Province, China) and authenticated by Xiujing Zhang (Beijing University of Chinese Medicine Third Affiliated Hospital). The voucher specimens (No. TTM-201802) were deposited at Beijing University of Chinese Medicine.

Take about $10 \mathrm{~kg}$ of air-dried plant materials, crushed into powder and through 60 mesh sizes. Soak the powder with $70 \%$ ethanol, heat and reflux for 3 hours with three times (1:8 for the first extraction and 1:6 for the second and third extraction, w/v for drug powder/ethanol solvent), then filtered. Next, the drug filtrates were evaporated and concentrated to remove ethanol under vacuum. Suspend the concentrated drug extract with distilled water and then add petroleum ether and n-butanol successively to partition, the n-butanol extract was considered as TST. Finally, collected the n-butanol extract, concentrated, vacuum dried, and finally got TST. The content of TST was $19.1 \mathrm{mg} / \mathrm{g}$ (based on crude drugs).

\section{Animals}

Forty-eight male Sprague Dawley (SD) rats with body weight $200 \pm 20 \mathrm{~g}$, were obtained from Beijing Vital River Laboratory Animal Technology Co., Ltd. (SCXK 2009-0011, Beijing, China). Six rats were kept in one polyacrylic cages. The rats were free access to food and water, housed in the standard controlled conditions with the temperature in $23 \pm 2^{\circ} \mathrm{C}$, the humidity in $50 \pm 5 \%$, and a $12 \mathrm{~h}$ day/night cycle. The rats were quarantined for one week before the experiments. The human care of the rats was according to the National Institutes of Health Guidelines of China and related ethical regulations of Beijing University of Chinese Medicine (Beijing, China).

\section{Experimental design}

All the rats were raised for one week before the formal experiment, in order to adapt the environment. The rats were randomly divided into four groups $(n=12)$ : Sham group, ischemia reperfusion injury (IR) group, TST-L $100 \mathrm{mg} / \mathrm{kg}$ group and TST-H $200 \mathrm{mg} / \mathrm{kg}$ group. The rats in TST group were oral administrated with the related dosage of TST for 14 days. The rats in Sham and IR group were oral administrated with normal saline for 14 days. After drug administration, the rats except Sham group were established IR model according to the method of Xu et al. reported [37]. The ischemic time is $30 \mathrm{~min}$, the reperfusion time is $120 \mathrm{~min}$. The rats in Sham group were surgically as IR rats except the suture was in the coronary artery without ligation. At the end of the experiment, all the rats were sacrificed by cervical dislocation.

\section{Hemodynamic indexes detection}


After myocardial ischemia and reperfusion, all the rats were detected the left ventricular systolic pressure (LVSP), left ventricular end diastolic pressure (LVDP), the maximum rate of left ventricular pressure rise $\left(+\mathrm{dp} / \mathrm{dt}_{\max }\right)$ and the maximum rate of decline $\left(-\mathrm{dp} / \mathrm{dt}_{\max }\right)$ by BL420F physiological instrument.

\section{Anti-oxidative index detection}

Heart homogenate $(10 \%, \mathrm{w} / \mathrm{v})$ was prepared by homogenizing the heart tissue in $150 \mathrm{mM}$ Tris-HCl buffered saline ( $\mathrm{pH} 7.2)$ with a polytron homogenizer. The enzyme activity of SOD and GSH-Px in heart tissue was measured according to the commercial kit. The data are read at the wavelength of $550 \mathrm{~nm}$ and expressed as $\mathrm{U} / \mathrm{L}$ and $\mathrm{nmol} / \mathrm{L}$ respectively. The lipid peroxidation products MDA in heart tissues was measured at $532 \mathrm{~nm}$ according to the protocol of commercial kit. The data of MDA was expressed as $\mu \mathrm{mol} / \mathrm{g}$ protein.

\section{Inflammatory factors detection}

The levels of TNF- $a$, IL-1 $\beta$ and IL- 6 in heart tissue homogenate were measured by ELISA kit from BioVision following the protocol provided by manufacture. The data were obtained at the wavelength of $450 \mathrm{~nm}$ and expressed as $\mathrm{pg} / \mathrm{mL}$. The preparation of heart tissue homogenate was followed the section of "Anti-oxidative index detection".

\section{Histopathological examination}

Heart specimens were fixed in $4 \%$ neutral formaldehyde buffer for overnight, and then embedded in paraffin, cut into $5 \mu \mathrm{m}$ thickness for hematoxylin and eosin (H\&E) staining. The H\&E sections were examined and photographed under a Olympus BX-50 light microscope at 200x magnification.

\section{TUNEL staining}

For terminal deoxynucleotidyl transferase-mediated dUTP nick-end labeling (TUNEL) staining, the hearts of rats were fixed in $4 \%$ paraform-aldehyde and dehydrated in $20 \%$ sucrose, embedded in paraffin and sectioned $(5 \mu \mathrm{m})$. The sections were treated as indicated in the situ cell death detection kit (Nanjingjiancheng, China). Thereafter, nuclei were co-stained with hematoxylin.

\section{Western blot}

Protein levels of caspase-3 (1:1000), caspase-9 (1:1000), caspase-12 (1:1000), AMPK (1:1000), p-AMPK (1:1000), acetylation NF-KB p65 (1:1000), and sirt1 (1:1000) in heart tissues were detected by Western blot. Heart tissue was washed with pre-cold PBS buffer, then homogenized with the pre-cold tissue homogenizer in homogenate buffer $(50 \mathrm{mmol} / \mathrm{L}, \mathrm{pH} 7.5 \mathrm{Tis}-\mathrm{HCl}, 150 \mathrm{mmol} / \mathrm{L} \mathrm{NaCl}, 1 \mathrm{mmol} / \mathrm{L}$ phenyl methyl sulfonyl fluoride, $1 \mathrm{mg} / \mathrm{mL}$ aprotinin, $4 \mathrm{mg} / \mathrm{mL}$ leupeptin) on ice bath. The homogenate centrifuge at $4^{\circ} \mathrm{C}, 10000 \mathrm{rpm} / \mathrm{min}$, then collected the supernatant, and detect the protein concentration by BCA kit. $40 \mu \mathrm{g}$ protein samples were loaded at SDS-PAGE electrophoresis gel for protein separation, then transferred the protein from gel to PVDF membrane at $4^{\circ} \mathrm{C}, 100 \mathrm{~V}$ for $1.5 \mathrm{~h}$. First antibody incubated 
overnight at $4^{\circ} \mathrm{C}$, second antibody incubated $1 \mathrm{~h}$ at room temperature, then the membrane was exposure and imaged at Bio-Rad imager (Bio-Rad ChemiDoc MP, USA).

\section{Statistical analysis}

The biological data presented as mean \pm SD. The statistical comparisons were made by One-way ANOVA test followed by Dunett's t-test with GraphPad Prism 6.0 statistical software. $P<0.05$ and $P<0.01$ showed a statistically significant.

\section{Results}

\section{Hemodynamic changes of rats in each group}

Compared with Sham group, the levels of LVSP and $+\mathrm{dp} / \mathrm{dt}_{\max }$ were significantly decreased, while the levels of LVDP and -dp/dt $\mathrm{max}_{\text {ax }}$ were significantly increased in the rats of IR group $(P<0.01$, Table 1$)$, this phenomenon exhibited that MIRI can damage the contractile and diastolic ability of myocardium. Compared with IR group, the levels of LVSP and $+\mathrm{dp} / \mathrm{dt}_{\max }$ were markedly increased, and the levels of LVDP and - $\mathrm{dp} / \mathrm{dt}_{\max }$ were significantly decreased in the rats of TST-treated groups $(P<0.05, P<0.01$, Table 1), which suggested that TST can effectively improve the hemodynamic indexes in IR rats.

Table 1

Hemodynamic changes of rats in each group

\begin{tabular}{|c|c|c|c|c|}
\hline Group & LVSP (mmHg) & LVDP (mmHg) & +dp/dtmax & $-\mathrm{dp} / \mathrm{dtmax}$ \\
\hline Sham & $168.35 \pm 15.02$ & $14.31 \pm 2.65$ & $8.89 \pm 1.02$ & $7.36 \pm 1.18$ \\
\hline IR & $126.63 \pm 10.31^{\# \#}$ & $7.16 \pm 1.38^{\# \#}$ & $4.74 \pm 0.73^{\# \#}$ & $4.85 \pm 0.74^{\# \#}$ \\
\hline TST-100 mg/kg & $142.17 \pm 11.58^{*}$ & $10.64 \pm 1.83^{*}$ & $6.13 \pm 1.11^{*}$ & $5.63 \pm 1.00^{\star}$ \\
\hline TST-200 mg/kg & $163.79 \pm 13.32^{\star \star}$ & $13.05 \pm 1.27^{\star \star}$ & $8.00 \pm 1.15^{\star \star}$ & $6.99 \pm 1.13^{\star *}$ \\
\hline
\end{tabular}

\section{Effects of TST on anti-oxidative indexes}

From Table 2, we can see that the levels of SOD and GSH-Px were significantly decreased in IR group, compared with Sham group $(P<0.01)$. Pre-treatment with 100 and $200 \mathrm{mg} / \mathrm{kg} \mathrm{TST}$, the levels of SOD and GSH-Px were significantly increased $(P<0.05, P<0.01)$, compared with IR group. The contents of MDA in heart tissue was significantly increased in IR group, compared with Sham group $(P<0.01)$. While the rats in TST pre-treated group were markedly decreased the contents of MDA, compared with that of IR group $(P<0.05, P<0.01)$. 
Table 2

Effect of TST on the levels of SOD, MDA and GSH-Px in heart tissue of rat

\begin{tabular}{|llll|}
\hline Group & SOD $(\mathrm{U} / \mathrm{L})$ & MDA $(\mu \mathrm{mol} / \mathrm{g})$ & GSH-Px $(\mathrm{nmol} / \mathrm{L})$ \\
\hline Sham & $77.59 \pm 7.02$ & $4.72 \pm 0.18$ & $18.62 \pm 2.07$ \\
\hline $\mathrm{IR}$ & $45.53 \pm 5.36^{\# \#}$ & $7.82 \pm 0.73^{\# \#}$ & $11.23 \pm 2.13^{\# \#}$ \\
\hline TST-100 mg/kg & $60.21 \pm 8.23^{*}$ & $6.05 \pm 1.13^{*}$ & $15.77 \pm 1.92^{*}$ \\
\hline TST-200 mg/kg & $70.59 \pm 8.37^{* *}$ & $5.13 \pm 1.01^{* *}$ & $17.95 \pm 2.25^{* *}$ \\
\hline $\begin{array}{l}\text { Data are expressed as mean } \pm \text { SD for each group ( } \mathrm{n}=12) .{ }^{* \#} p<0.01 \text { vs sham group }{ }^{*} p<0.05{ }^{* *} p< \\
\text { 0.01 vs IR group. IR: ischemia-reperfusion group, TST: total Saponins from Trillium Tschonoskii } \\
\text { Maxim. }\end{array}$ \\
\hline
\end{tabular}

\section{Effect Of Tst On Macrophage Infiltration}

In Fig. 1, macrophages in heart tissue are dark brown. In the sham operation group, there were a few macrophages in the heart tissue, while in IR model group, there were a lot of macrophage infiltration. Compared with IR model group, the number of macrophages were significantly decreased in the heart tissue of low-dose TST-L rats, while only a small amount of macrophages were infiltrated in the heart tissue of high-dose TST-H rats.

\section{Effects of TST on inflammatory factors}

The inflammation of the heart tissue were evaluated by the levels of TNF- $a, \mathrm{IL}-1 \beta$ and IL- 6 . Compared with Sham group, the levels of TNF- $a$, IL- $1 \beta$ and IL- 6 were remarkably increased in IR group $(P<0.01$, Table 3$)$. TST pre-treated group were significantly decreased the levels of TNF- $a$, IL- $1 \beta$ and IL-6, compared with IR group $(P<0.05, P<0.01$, Table 3$)$.

Table 3

Effect of TST on the levels of TNF- $a$, IL-1 $\beta$ and IL- 6 in heart tissue of rat

\begin{tabular}{|c|c|c|c|}
\hline Group & TNF- $a(\mathrm{pg} / \mathrm{mL})$ & $\mathrm{IL}-1 \beta(\mathrm{pg} / \mathrm{mL})$ & IL-6 (pg/mL) \\
\hline Sham & $96.79 \pm 2.52$ & $13.16 \pm 3.27$ & $35.27 \pm 6.07$ \\
\hline IR & $200.63 \pm 22.21^{\# \#}$ & $159.36 \pm 15.34^{\# \#}$ & $141.31 \pm 22.45^{\# \#}$ \\
\hline TST-100 mg $/ \mathrm{kg}$ & $133.84 \pm 10.12^{*}$ & $67.93 \pm 5.03^{*}$ & $71.03 \pm 7.13^{*}$ \\
\hline TST-200 mg/kg & $98.57 \pm 6.65^{\star \star}$ & $33.51 \pm 3.95^{\star \star}$ & $40.17 \pm 5.05^{\star \star}$ \\
\hline
\end{tabular}




\section{Discussion}

Myocardial ischemic disease is one of the main diseases endangering human health[38]. The clinical therapy method of thrombolysis, percutaneous coronary angioplasty, and coronary artery bypass grafting can restore blood supply and improve heart function in a short time, but still cannot avoid the aggravation of tissue damage during ischemia-reperfusion, or even lead to irreversible function and organic damage[39]. The mechanism of MIRI is currently considered to be related to the role of free radicals, intracellular calcium overload, leukocyte activation, endoplasmic reticulum stress and mitochondrial dysfunction. Among them, reactive oxygen species (ROS) damage is considered to be important for MIRI[40]. Many studies have shown that excessive release of oxygen free radicals can induce irreversible pathological changes of myocardial cells, thus aggravating the reperfusion injury of blood flow[41]. In this study, through the pathological observation of myocardial tissue in the model group, we found that the necrosis and edema of myocardial tissue make the inflammatory infiltration of myocardial cells.

In this study, the levels of LVSP and $+d p / d t_{\max }$ in IR group were significantly increased, LVDP and $\mathrm{dp} / \mathrm{dt}_{\max }$ were markedly decreased, compared with Sham group, which indicating that MIR can lead to myocardial necrosis. While the myocardial hemodynamics of the rats pre-treated with TST were significantly improved, indicating that TST can improve and protect the myocardial structure and function, improve the pathological changes and inhibit the MIRI.

In oxidative stress, MDA can indicate the degree of lipid peroxidation and the degree of damage of oxygen free radicals to cardiomyocytes, while SOD and GSH-Px can clear these oxygen free radicals and degrade them. Therefore, the activity of SOD and GSH-Px shows the ability of myocardial cells to scavenge oxygen free radicals[42, 43]. In the study, the activity of SOD and GSH-Px were significantly decreased, while the contents of MDA was markedly increased in IR group. TST pre-treatment can significantly increase the enzyme activity of SOD and GSH-Px, and significantly decrease the MDA products. This results exhibited that TST can increase the anti-oxidative ability of cardiomyocytes, further to protect the MIRI rats, decrease the injury.

In addition, in the study, we detected the protein expression of caspase-12, caspase-9, and caspase-3 in classical signaling pathway of apoptosis by western blot method. The result showed that TST treatment group significantly reduced the apoptosis protein expression, compared with the IR group. This result indicated that TST can reduce the number of myocardial apoptosis, which can nourish the myocardium, further to enhance the cardiac function of ischemia-reperfusion rats, and improve the hemodynamics in some degree.

Furthermore, AMPK, as an important protein kinase in the process of cell energy metabolism, can regulate Sirt 1 activity and reduce the expression of inflammatory genes by activating AMPK, thus inhibiting the inflammatory response[44, 45]. In addition, the reported study showed that TST can protect the acute liver injury by inhibiting NF-KB expression[46, 47]. More importantly, it was reported that the activation of 
AMPK/Sirt1 could inhibited MIRI through alleviate inflammation reaction[32-35], which is very important for MIRI therapy in clinic. Thus, in this study, we further evaluated that whether TST plays an antiinflammatory role by stimulating AMPK/Sirt1/NF- $K B$ signaling pathway[48]. The result showed that TST pre-treatment can increase the protein expression of $p$-AMPK and Sirt1, decrease the protein expression of acetylation NF- $K B$ p65, compared with the IR group, which indicated that TST exerts the protective effect in MIRI rats may be through activation the signaling pathway of AMPK/Sirt1/NF- $K B$. Since the current study has many limitations, the study assessed only the effect of TST when it is pre-treated to the animals, the effectiveness of TST is to examine the long-term effects of TST treatment to cardiac function in rat models of IMRI or heart failure is a future direction for the work.

\section{Conclusion}

In summary, TST can inhibit inflammatory cell infiltration during MIRI, reduce the number of myocardial cell apoptosis, enhance cardiac function, and regulate the protein expression of $p$-AMPK and Sirt1, which further to improve hemodynamics, ensure blood perfusion and nutrition supply for the demand of surrounding tissues. The study will help to clarify the mechanism of MIRI and provide a new possible therapy method for the clinical patients.

\section{Abbreviations}

TST, total saponins from Trillium Tschonoskii Maxim.; TTM, Trillium Tschonoskii Maxim.; IRI, ischemia reperfusion injury; IR, ischemia-reperfusion; MIRI, myocardial ischemia reperfusion injury; CVDs, cardiovascular diseases; SD, sprague Dawley; TNF- $a$, tumor necrosis factor-alpha; IL-6, interleukin-6; SOD, superoxide dismutase; GSH-Px, glutathione peroxidase; MDA, malondialdehyde; HRP, horseradish peroxidase; LVSP, left ventricular systolic pressure; LVDP, left ventricular end diastolic pressure; $+\mathrm{dp} / \mathrm{dt}_{\mathrm{max}}$ the maximum rate of left ventricular pressure rise; - $\mathrm{dp} / \mathrm{dt}_{\max }$, the maximum rate of decline.

\section{Declarations}

\section{Ethics approval and consent to participate}

All experiments related animals in the manuscript were reviewed and approved by the Institutional Animal Care and Use Committee on the Ethics of Animal Experiments of Beijing University of Chinese Medicine (No. BUCM-4-2018031704-1023).

\section{Consent to publish}

Not applicable.

\section{Availability of data and materials}


All data generated or analyzed during this study can be obtained from the corresponding Author with a reasonable request.

\section{Competing Interests}

The authors declare that they have no competing interests.

\section{Funding}

The study was funded by Beijing University of Chinese Medicine.

\section{Authors' contributions}

WW and XZ designed and guaranteed the whole experiment studies; WW, JT, QX and DM carried out all the experiments; WW, JT, QX and DM analyzed the statistical data, WW drafted the manuscript; XZ edited and revised the whole manuscript; all authors read and approved the final manuscript.

\section{Acknowledgements}

We thank the animal care and the technical method guide by the workers in the animal facility of Beijing Vital River Laboratory Animal Technology Co., Ltd., China.

\section{References}

1. World. Health Organization: https://www.who.int/en/news-room/fact-sheets/detail/cardiovasculardiseases-(cvds) 2016.

2. Zhou SS, Jin JP, Wang JQ, Zhang ZG, Freedman JH, Zheng Y, Cai L. miRNAS in cardiovascular diseases: potential biomarkers, therapeutic targets and challenges. Acta pharmacologica Sinica. 2018;39(7):1073-84.

3. Kim ESH, Beckman JA. Introduction to the Vascular Medicine Issue of Progress in Cardiovascular Diseases. Prog Cardiovasc Dis. 2018;60(6):565-6.

4. Wu MY, Yiang GT, Liao WT, Tsai AP, Cheng YL, Cheng PW, Li CY, Li CJ. Current Mechanistic Concepts in Ischemia and Reperfusion Injury. Cellular physiology biochemistry: international journal of experimental cellular physiology biochemistry pharmacology. 2018;46(4):1650-67.

5. Binder A, Ali A, Chawla R, Aziz HA, Abbate A, Jovin IS. Myocardial protection from ischemiareperfusion injury post coronary revascularization. Expert Rev Cardiovasc Ther. 2015;13(9):1045-57.

6. Kezic A, Stajic N, Thaiss F. Innate Immune Response in Kidney Ischemia/Reperfusion Injury: Potential Target for Therapy. Journal of immunology research 2017, 2017:6305439.

7. Wu D, Wang J, Wang H, Ji A, Li Y. Protective roles of bioactive peptides during ischemia-reperfusion injury: From bench to bedside. Life sciences. 2017;180:83-92.

8. Mokhtari-Zaer A, Marefati N, Atkin SL, Butler AE, Sahebkar A. The protective role of curcumin in myocardial ischemia-reperfusion injury. Journal of cellular physiology. 2018;234(1):214-22. 
9. Lu Y, Feng Y, Liu D, Zhang Z, Gao K, Zhang W, Tang H. Thymoquinone Attenuates Myocardial Ischemia/Reperfusion Injury Through Activation of SIRT1 Signaling. Cellular physiology biochemistry: international journal of experimental cellular physiology biochemistry pharmacology. 2018;47(3):1193-206.

10. Tang L, Mo Y, Li Y, Zhong Y, He S, Zhang Y, Tang Y, Fu S, Wang X, Chen A. Urolithin A alleviates myocardial ischemia/reperfusion injury via PI3K/Akt pathway. Biochem Biophys Res Commun. 2017;486(3):774-80.

11. Wang L, Lin R, Guo L, Hong M: Rosuvastatin relieves myocardial ischemia/reperfusion injury by upregulating PPARgamma and UCP2. Molecular medicine reports 2018, 18(1):789-798.

12. Wang BF, Yoshioka J. The Emerging Role of Thioredoxin-Interacting Protein in Myocardial Ischemia/Reperfusion Injury. J Cardiovasc Pharmacol Therap. 2017;22(3):219-29.

13. Xie B, Liu X, Yang J, Cheng J, Gu J, Xue S. PIAS1 protects against myocardial ischemia-reperfusion injury by stimulating PPARgamma SUMOylation. BMC Cell Biol. 2018;19(1):24.

14. Tire Y, Sarkilar G, Esen H, Onoglu R, Uzun ST. The effect of intrathecal sufentanil preconditioning against myocardial ischemia-reperfusion injury. Bratislavske lekarske listy. 2018;119(4):240-4.

15. O'Shea KM, Ananthakrishnan R, Li Q, Quadri N, Thiagarajan D, Sreejit G, Wang L, Zirpoli H, Aranda JF, Alberts AS, et al: The Formin, DIAPH1, is a Key Modulator of Myocardial Ischemia/Reperfusion Injury. EBioMedicine 2017, 26:165-174.

16. Hu C, Tian Y, Xu H, Pan B, Terpstra EM, Wu P, Wang H, Li F, Liu J, Wang X. Inadequate ubiquitinationproteasome coupling contributes to myocardial ischemia-reperfusion injury. J Clin Investig. 2018;128(12):5294-306.

17. Dasagrandhi D, Muthuswamy RASK, Lennox A, Jayavelu AM, Devanathan T, Kesavan Swaminathan V. J: Ischemia/reperfusion injury in male guinea pigs: An efficient model to investigate myocardial damage in cardiovascular complications. Biomedicine pharmacotherapy = Biomedecine pharmacotherapie. 2018;99:469-79.

18. Tong S, Zhang L, Joseph J, Jiang X: Celastrol pretreatment attenuates rat myocardial ischemia/ reperfusion injury by inhibiting high mobility group box 1 protein expression via the PI3K/Akt pathway. Biochemical and biophysical research communications 2018, 497(3):843-849.

19. Thind GS, Agrawal PR, Hirsh B, Saravolatz L, Chen-Scarabelli C, Narula J, Scarabelli TM. Mechanisms of myocardial ischemia-reperfusion injury and the cytoprotective role of minocycline: scope and limitations. Future cardiology. 2015;11(1):61-76.

20. Luo HB, Shang N, Xie WZ, Wen DJ, Qu M, Huang S, Fan SS, Chen W, Mou NQ, Liu XY, et al. Trillium tschonoskii maxim extract attenuates abnormal Tau phosphorylation. Neural regeneration research. 2018;13(5):915-22.

21. Wang L, Du J, Zhao F, Chen Z, Chang J, Qin F, Wang Z, Wang F, Chen X, Chen N. Trillium tschonoskii maxim saponin mitigates $\mathrm{D}$-galactose-induced brain aging of rats through rescuing dysfunctional autophagy mediated by Rheb-mTOR signal pathway. Biomedicine pharmacotherapy = Biomedecine pharmacotherapie. 2018;98:516-22. 
22. Wu H, Qiu Y, Shu Z, Zhang X, Li R, Liu S, Chen L, Liu H, Chen N. Protective effect of Trillium tschonoskii saponin on CCl4-induced acute liver injury of rats through apoptosis inhibition. Can $\mathrm{J}$ Physiol Pharmacol. 2016;94(12):1291-7.

23. Teng JF, Qin DL, Mei QB, Qiu WQ, Pan R, Xiong R, Zhao Y, Law BY, Wong VK, Tang Y, et al. Polyphyllin VI, a saponin from Trillium tschonoskii Maxim. induces apoptotic and autophagic cell death via the ROS triggered mTOR signaling pathway in non-small cell lung cancer. Pharmacological research. 2019;147:104396.

24. Huang W, Zou K, Xiong B. The rhizome of Trillium tschonoskii Maxim. extract induces apoptosis in human lung cancer cells. Zeitschrift fur Naturforschung C Journal of biosciences. 2011;66(910):477-84.

25. Wang FJ, Wang KK, Chen XB, Liu JH, Gao S, Liu X. [Intervention effect of trillium tschonoskii maxim extract solution on myocardial injury in diabetic rats]. Zhongguo ying yong sheng li xue za zhi= Zhongguo yingyong shenglixue zazhi = Chinese. journal of applied physiology. 2016;32(2):177-80.

26. Zhan GJ, Xiao BJ, Yang NA. [The protective effects and mechanisms of Trillium tschonoskii Maxim on rats' liver damage induced by lipopolysaccharide]. Zhongguo ying yong sheng li xue za zhi = Zhongguo yingyong shenglixue zazhi = Chinese. journal of applied physiology. 2016;32(4):373-7.

27. Huang W, Zou K. Cytotoxicity of a plant steroidal saponin on human lung cancer cells. Asian Pacific journal of cancer prevention: APJCP. 2011;12(2):513-7.

28. Gao X, Sun W, Fu Q, Niu X. Rapid Identification of Steroidal Saponins in Trillium tschonoskii Maxim by Ultraperformance Liquid Chromatography Coupled to Electrospray Ionisation Quadrupole Time-ofFlight Tandem Mass Spectrometry. Phytochem Anal. 2015;26(4):269-78.

29. Li D, Liu H, Ni W, Xiao WL, He L, Guo ZY, Qin XJ, Liu HY. Molecular networking-based strategy for the discovery of polyacetylated 18-norspirostanol saponins from Trillium tschonoskii maxim. Phytochemistry. 2019;168:112125.

30. Yan T, Yu X, Sun X, Meng D, Jia JM. A new steroidal saponin, furotrilliumoside from Trillium tschonoskii inhibits lipopolysaccharide-induced inflammation in Raw264.7 cells by targeting PI3K/Akt, MARK and Nrf2/HO-1 pathways. Fitoterapia. 2016;115:37-45.

31. Qian S, Tong S, Wu J, Tian L, Qi Z, Chen B, Zhu D, Zhang Y. Paris saponin VII extracted from Trillium tschonoskii induces autophagy and apoptosis in NSCLC cells. J Ethnopharmacol. 2020;248:112304.

32. Tian L, Cao W, Yue R, Yuan Y, Guo X, Qin D, Xing J, Wang X. Pretreatment with Tilianin improves mitochondrial energy metabolism and oxidative stress in rats with myocardial ischemia/reperfusion injury via AMPK/SIRT1/PGC-1 alpha signaling pathway. J Pharmacol Sci. 2019;139(4):352-60.

33. Qiu L, Luo Y, Chen X. Quercetin attenuates mitochondrial dysfunction and biogenesis via upregulated AMPK/SIRT1 signaling pathway in OA rats. Biomedicine pharmacotherapy = Biomedecine pharmacotherapie. 2018;103:1585-91.

34. Potenza MA, Sgarra L, Nacci C, Leo V, De Salvia MA, Montagnani M. Activation of AMPK/SIRT1 axis is required for adiponectin-mediated preconditioning on myocardial ischemia-reperfusion (I/R) injury in rats. PloS one. 2019;14(1):e0210654. 
35. Jing HR, Luo FW, Liu XM, Tian XF, Zhou Y. Fish oil alleviates liver injury induced by intestinal ischemia/reperfusion via AMPK/SIRT-1/autophagy pathway. World journal of gastroenterology. 2018;24(7):833-43.

36. Li M, Ouyang J, Zhang Y, Cheng BCY, Zhan Y, Yang L, Zou H, Zhao H. Effects of total saponins from Trillium tschonoskii rhizome on grey and white matter injury evaluated by quantitative multiparametric MRI in a rat model of ischemic stroke. J Ethnopharmacol. 2018;215:199-209.

37. Xu Z, McElhanon KE, Beck EX, Weisleder N. A Murine Model of Myocardial Ischemia-Reperfusion Injury. Methods in molecular biology. 2018;1717:145-53.

38. Akbalaeva BA, Batyraliev TA, Fettzer DV, Sidorenko BA. [lschemic Heart Disease: Focus on Acute Infarction of the Right Ventricular Myocardium]. Kardiologiia 2017(1):90-94.

39. Cao DJ, Schiattarella GG, Villalobos E, Jiang N, May HI, Li T, Chen ZJ, Gillette TG, Hill JA. Cytosolic DNA Sensing Promotes Macrophage Transformation and Governs Myocardial Ischemic Injury. Circulation. 2018;137(24):2613-34.

40. Thosar SS, Butler MP, Shea SA. Role of the circadian system in cardiovascular disease. J Clin Investig. 2018;128(6):2157-67.

41. Ding S, Fan Z, Lin C, Dai Q, Zhou J, Huang H, Xu Y, Zhong C. Therapeutic Effects of IschemicPreconditioned Exosomes in Cardiovascular Diseases. Adv Exp Med Biol. 2017;998:271-81.

42. Chen J, Ceholski DK, Turnbull IC, Liang L, Hajjar RJ. Ischemic Model of Heart Failure in Rats and Mice. Methods in molecular biology. 2018;1816:175-82.

43. Pennella S, Reggiani Bonetti L, Migaldi M, Manenti A, Lonardi R, Giuliani E, Barbieri A, Farinetti A, Mattioli AV. Does stem cell therapy induce myocardial neoangiogenesis? Histological evaluation in an ischemia/reperfusion animal model. Journal of cardiovascular medicine. 2017;18(4):277-82.

44. Thirupathi A, de Souza CT. Multi-regulatory network of ROS: the interconnection of ROS, PGC-1 alpha, and AMPK-SIRT1 during exercise. Journal of physiology biochemistry. 2017;73(4):487-94.

45. Gu X, Cai Z, Cai M, Liu K, Liu D, Zhang Q, Tan J, Ma Q. AMPK/SIRT1/p38 MAPK signaling pathway regulates alcoholinduced neurodegeneration by resveratrol. Mol Med Rep. 2018;17(4):5402-8.

46. Zhang J, Zhang SD, Wang P, Guo N, Wang W, Yao LP, Yang Q, Efferth T, Jiao J, Fu YJ. Pinolenic acid ameliorates oleic acid-induced lipogenesis and oxidative stress via AMPK/SIRT1 signaling pathway in HepG2 cells. Eur J Pharmacol. 2019;861:172618.

47. Keshk WA, Soliman NA, Ali DA, Elseady WS. Mechanistic evaluation of AMPK/SIRT1/FXR signaling axis, inflammation, and redox status in thioacetamide-induced liver cirrhosis: The role of Cichorium intybus linn (chicory)-supplemented diet. J Food Biochem. 2019;43(8):e12938.

48. Song YN, Yuan D, Zhang CC, Wang LP, He YM, Wang T, Zhou ZY. [Effect of saponins extracted from Panax japonicus on inhibiting cardiomyocyte apoptosis by AMPK/Sirt1/NF-kappaB signaling pathway in aging rats]. Zhongguo Zhong yao za zhi $=$ Zhongguo zhongyao zazhi $=$ China journal of Chinese materia medica. 2017;42(23):4656-60.

\section{Figures}




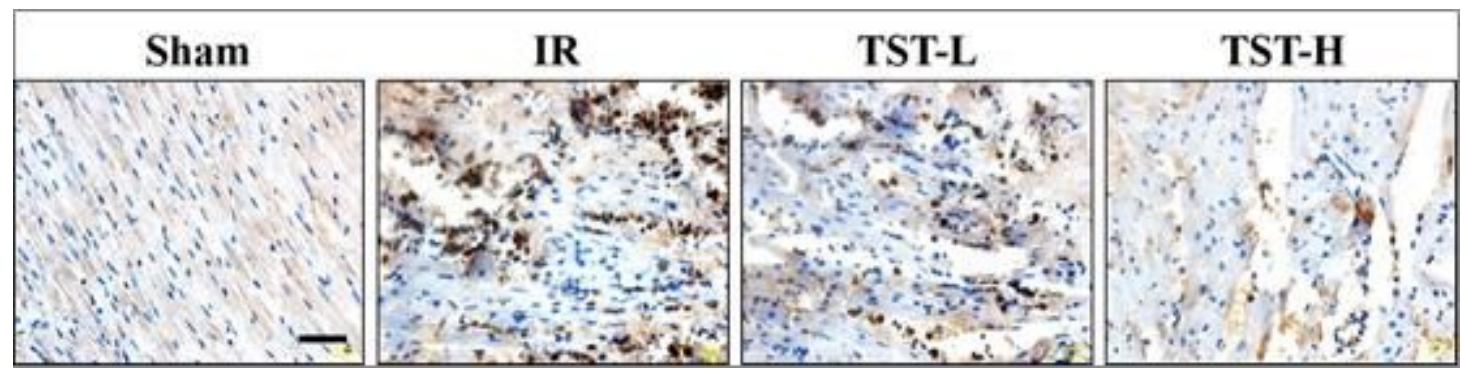

Figure 1

Distribution of macrophages in heart tissue of rats in each group. Scale bar $200 \mu \mathrm{m}$.

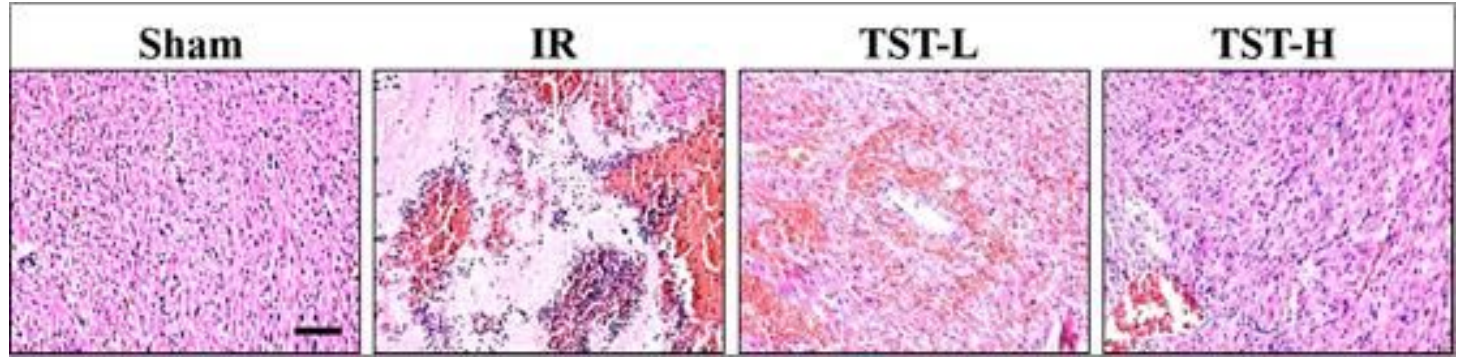

Figure 2

Histopathological changes of myocardium in each groups by H \& E staining. Scale bar $200 \mu \mathrm{m}$.

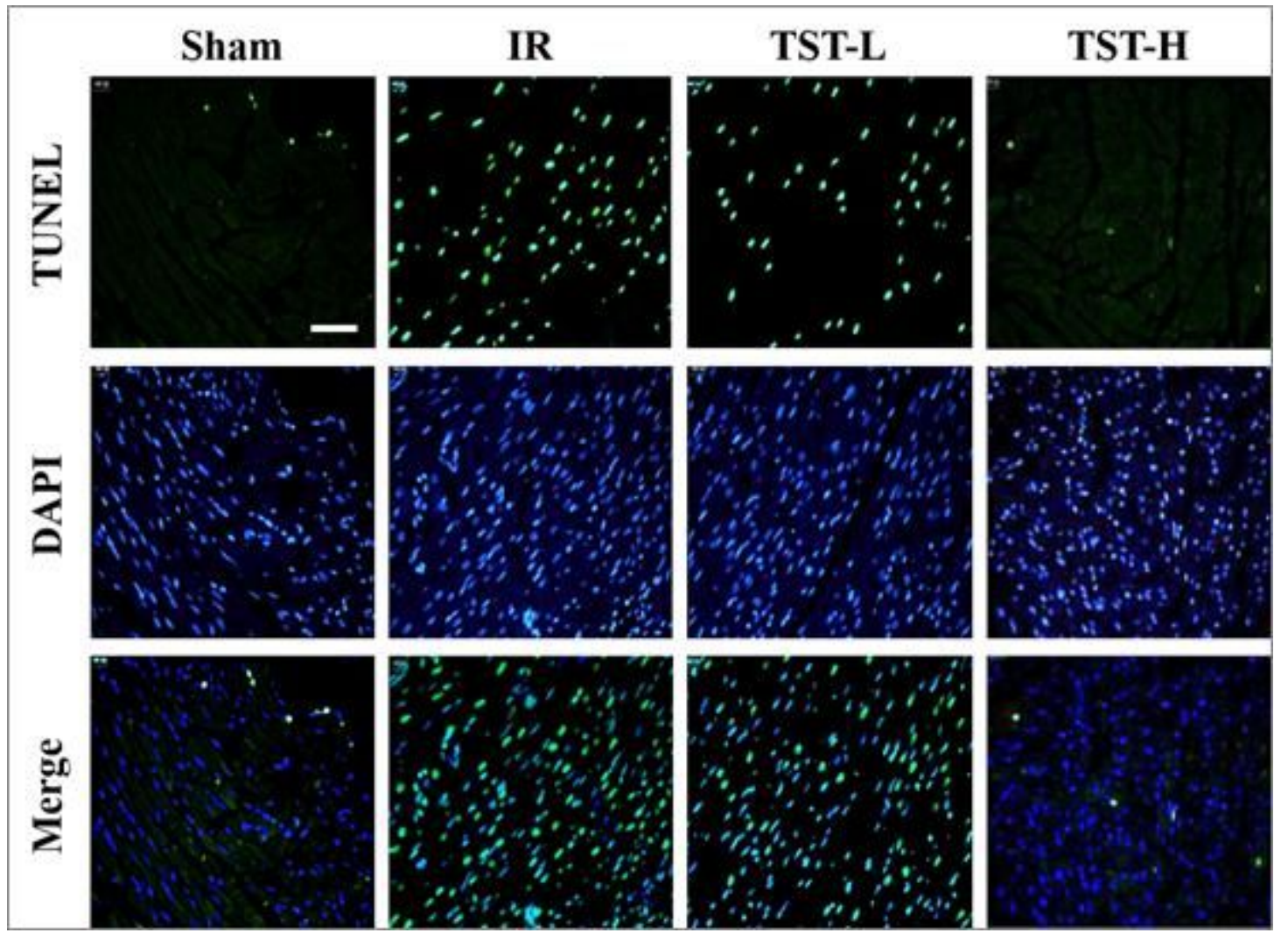

Figure 3

TUNEL staining for the apoptosis of cardiomyocyte in each group. Scale bar $200 \mu \mathrm{m}$. 


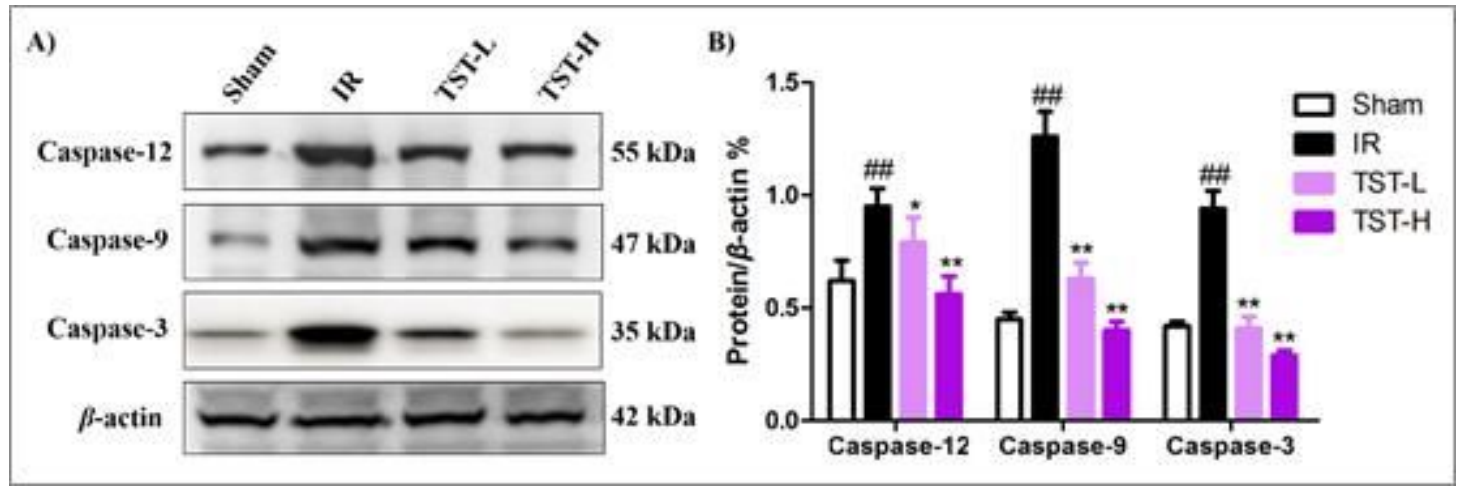

\section{Figure 4}

The protein expression of apoptosis proteins in myocardium tissue of all group rats. A. Protein expression of caspase 3,9 , and 12 in myocardium tissue by western blot. B. Quantitative analysis of the protein expression of caspase 3,9 , and 12 . The image showed a representative cropping blots. \#\#P $<0.01$ vs Sham group, $* \mathrm{P}<0.05,{ }^{*} \mathrm{P}<0.01$ vs IR group.

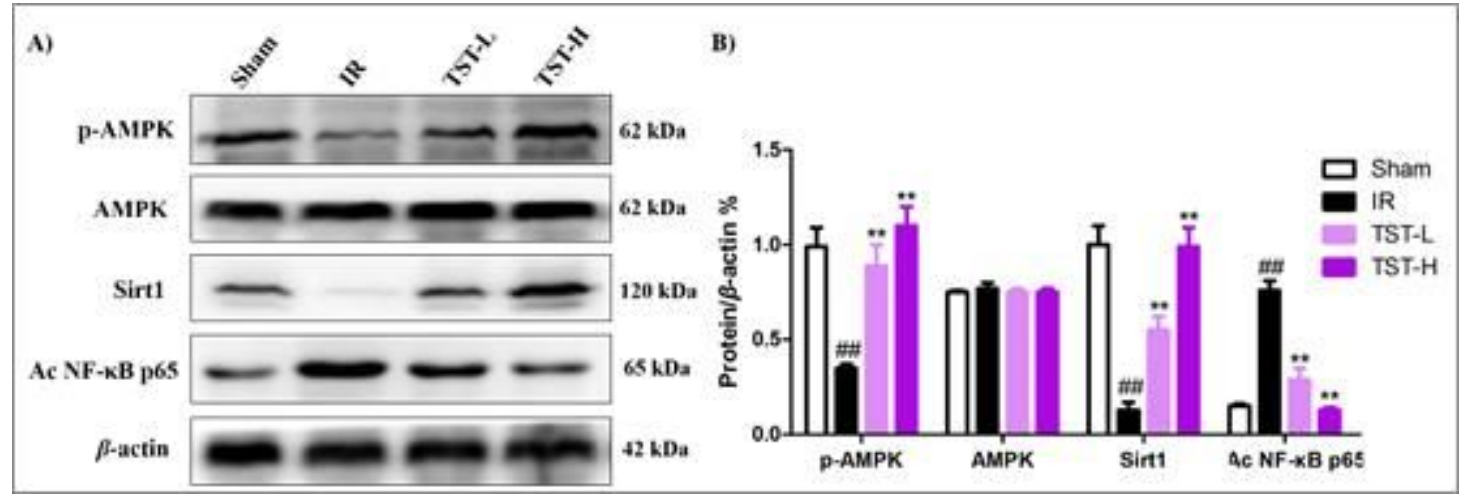

\section{Figure 5}

The protein expression of AMPK/Sirt1 signaling pathway in myocardium tissue of all group rats by western blot. A. The image showed a representative cropping blots. B. Quantitative analysis of the protein expression of p-AMPK, Sirt1 and Ac NF-KB p65. The image showed a representative cropping blots. \#\#P< 0.01 vs Sham group, ${ }^{*} P<0.05,{ }^{\star *} \mathrm{P}<0.01$ vs IR group. 